\title{
Effects of Aging and Different Mechanical Recycling Processes on the Structure and Properties of Poly(lactic acid)-clay Nanocomposites
}

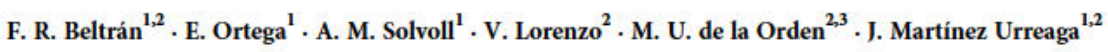

\begin{abstract}
The growing use of poly(lactic acid) (PLA) and PLA-based nanocomposites in packaging has raised the interest of studying the mechanical recycling of the wastes and the properties of the recycled materials. The main objective of this work was to study the effect of two different mechanical recycling processes on the structure and properties of a PLA-montmorillonite nanocomposite. The two recycling processes included accelerated thermal and photochemical aging steps to simulate the degradation experienced by post-consumer plastics during their service life. One of them also included a demanding washing process prior to the reprocessing. A decrease in the molecular weight of PLA was observed in the recycled materials, especially in those subjected to the washing step, which explained the small decrease in microhardness and the increased water uptake at long immersion times. Water absorption at short immersion times was similar in virgin and recycled materials and was accurately described using a Fickian model. The recycled materials showed increased thermal, optical and
\end{abstract}

Data availability: The datasets analyzed during the current study are available from the corresponding author on reasonable request.

\section{F. R. Beltrán}

f.beltran@upm.es

1 Departamento de Ingeniería Química Industrial y del Medio Ambiente, E.T.S.I. Industriales, Universidad Politécnica de Madrid, José Gutiérrez Abascal 2, 28006 Madrid, Spain

2 Grupo de Investigación "Polímeros: Caracterización y Aplicaciones (POLCA)", E.T.S.I. Industriales, Universidad Politécnica de Madrid, José Gutiérrez Abascal 2, 28006 Madrid, Spain

3 Departamento de Química Orgánica I, Facultad de Óptica y Optometría, Universidad Complutense de Madrid, Arcos de Jalón 118, 28037 Madrid, Spain gas barrier properties due to the improved clay dispersion that was observed by XRD and TEM analysis. The results suggest that recycled PLA-clay nanocomposites can be used in demanding applications.

Keywords Mechanical recycling $\cdot$ Poly(lactic acid $)$. Nanocomposites · Structure $\cdot$ Properties

\section{Introduction}

Poly(lactic acid) (PLA) represents an interesting alternative to the fossil-fuel based polymers in packaging applications, especially for food products. This is due to PLA being considered as biodegradable, safe in food contact and comparable to some commodity plastics, such as polyethylene terephthalate (PET), in optical and mechanical properties [1-3]. However, other PLA properties, such as impact strength and gas permeability, show only moderate values and therefore the addition of different nanometric reinforcing agents, such as organically modified clays, has been studied over the past years. The nanocomposites obtained show, in general, improved impact strength, fire resistance and gas barrier properties [4-6]. These characteristics have placed PLA as one of the most important bioplastics in the market, with a global production capacity of 205,000 tons in 2014, which is expected to exceed 500,000 tons in 2020 [7].

The expected growth in production of PLA could generate some environmental and social issues, especially if the origin of the raw materials necessary for its production is taken into account. PLA is produced by the ring opening polymerization of lactide, the cyclic dimer of lactic acid, which is obtained by the fermentation of the glucose present in some food products such as corn, potato or sugar beet [8]. This situation could cause an increasing demand of 
large amounts of land to produce such food products, also increasing the environmental impact of the manufacture of PLA [9]. Furthermore, some farmers could be tempted to exchange the cheap food production for the crops used in the production of PLA, compromising the health and sustenance of developing countries [10]. Lastly, there are some concerns regarding the end-of-life scenarios for this polymer, since they play a very important role on the environmental impact of PLA. Some studies, such as those conducted by Piemonte [11], Cosate de Andrade et al. [12] and Rossi et al. [13] point out that composting is not necessarily the best alternative in the case of PLA, suggesting that mechanical recycling might be a more interesting alternative. This is especially important in the commercial grades used in packaging applications, since they degrade at a slower rate than the accumulation of wastes [14-16].

Considering the social and environmental issues derived from the massive production of PLA and PLA-based materials, it can be concluded that mechanical recycling is an interesting end-of-life scenario for PLA wastes coming from packaging applications, since it allows the reduction in raw materials, energy and emissions, thus decreasing the environmental impact of the use of PLA. However, in order to evaluate the feasibility of the mechanical recycling, it is necessary to compare the properties of virgin and recycled materials, since if there is an important decrease in the properties of the recycled materials, the mechanical recycling would be unfeasible.

Despite the clear interest of the mechanical recycling, the properties of recycled PLA and PLA-based materials have been scarcely studied up to the present. Different authors have measured the thermal and mechanical properties of neat PLA after several reprocessing steps. For instance, Scaffaro et al. [17], Badía et al. [18] and Żenkiewicz et al. [19] reported that PLA presented reduced mechanical properties after 1,5 and 10 reprocessing cycles. However, Nascimento et al. [20] pointed out that a single reprocessing step does not significantly affect the structure, thermal and mechanical properties of PLA.

In general, these studies do not consider the degradation (thermal, photochemical and hydrolytic) of the PLA during its useful life nor do stages of accelerated aging, although the degradation of the polymer prior to reprocessing can play a very important role in the decrease of the properties of the recycled plastic. In a previous work we have studied the hydrolytic degradation of PLA subjected to different recycling processes that include, in addition to the reprocessing step, accelerated aging and washing steps to simulate the degradation of the material during the service life and the cleaning of a plastic waste coming from food packaging, respectively [21]. The results revealed that the recycled plastics show good resistance against the hydrolytic degradation, although the behavior of the recycled materials depends on the conditions of the recycling process. The impact of the different mechanical recycling processes on the mechanical, optical, thermal and barrier properties of the recycled plastic was also limited [1].

As it has been mentioned, the mechanical recycling of unfilled PLA has already been studied and reported in the literature. However, the behavior of PLA based nanocomposites does not have to be the same, since the presence of clays could promote the degradation of the polymer during its service life and also during the recycling processes, thus affecting the final properties of the recycled materials. Despite the growing use of clays in PLA based materials, in order to improve the properties of the polymer, there is little data available regarding the behavior of mechanically recycled PLA nanocomposites. In this regard, Kozlowski and Macyszyn studied the reprocessing of a PLA-montmorillonite nanocomposite and found that PLA suffered increasing degradation during the successive reprocessing cycles, which leaded to higher values of the melt flow rate. However, oxygen permeability was reduced as a result of the reprocessing [22]. In the same vein, Scaffaro et al. studied the effect of five reprocessing cycles in nanocomposites of PLA and hydrotalcites. They reported that reprocessing caused an improvement of the dispersion of the clay particles, which led to an increase of the mechanical properties in the first three reprocessing steps. However, after four and five reprocessing cycles, the degradation of PLA was severe, thus decreasing the performance of the material [23]. In both cited works, the degradation of the material during its service life and possible washing steps were not considered.

Consequently, the main aim of this work was to study the effect of different mechanical recycling processes, including accelerated aging stages, on the properties of PLA-clay nanocomposites. A commercial grade of PLA, especially designed for packaging applications, and $2 \mathrm{wt} \%$ of an organically modified montmorillonite were melt compounded and compression molded into films. A portion of these films were subjected to two different mechanical recycling processes, one comprising an accelerated thermal and photochemical aging before the second extrusion and compression molding step, and other including a demanding washing step between the accelerated aging and the reprocessing step. The effect of both processes on the structure and properties of the material was followed by water absorption measurements, dilute solution viscosimetry (IV), infrared spectroscopy (FTIR-ATR), X-ray diffraction (XRD), transmission electron microscopy (TEM), differential scanning calorimetry (DSC), thermogravimetric analysis (TGA), ultraviolet-visible spectroscopy (UV-Vis) and microhardness measurements. The results indicate that, although the recycling processes modify the structure of the nanocomposites, the recycled materials retain, to a great extent, the properties of the virgin material. 


\section{Materials and Methods}

\section{Materials and Preparation of the Samples}

The PLA used was a commercial grade designed for packaging (Ingeo 2003D, Natureworks ${ }^{\mathrm{TM}}$ ), with a melt mass-flow rate of $6 \mathrm{~g} / 10 \mathrm{~min}\left(2.16 \mathrm{~kg}\right.$ at $\left.210{ }^{\circ} \mathrm{C}\right)$. Prior to processing, the material was dried according to the manufacturer's recommendations $\left(20 \mathrm{~min}\right.$ at $100{ }^{\circ} \mathrm{C}$ and $2 \mathrm{~h}$ in a vacuum oven at $85^{\circ} \mathrm{C}$ ). The clay used was Cloisite ${ }^{\mathrm{TM}}$ 30B (C30), an organically modified montmorillonite supplied by Southern Clay Products (USA), where the organic modifier is a methyl, dihydroxyethyl, dehydrogenated tallow, quaternary ammonium chloride. The clay was dried in a convection oven at $100{ }^{\circ} \mathrm{C}$ for $2 \mathrm{~h}$.

Neat PLA and the PLA nanocomposite with $2 \mathrm{wt} \%$ C30 were melt compounded in a Rondol Microlab twin-screw microcompounder, with $\mathrm{L} / \mathrm{D}=20$, at $60 \mathrm{rpm}$. The barrel temperature profile from hopper to die was $125,160,190$, $190,180{ }^{\circ} \mathrm{C}$. The films with a thickness of $230 \pm 10 \mu \mathrm{m}$ were obtained by compression in an IQAP-LAP hot-plate press at $190{ }^{\circ} \mathrm{C}$, beginning with a melting step, with no pressure, for $5 \mathrm{~min}$, followed by a degasification step for $2 \mathrm{~min}$ and cooling between cold plates at $14 \mathrm{MPa}$ for $5 \mathrm{~min}$.

\section{Recycled Materials}

In order to simulate the degradation of the material during its service life, the films of the virgin polymer were first subjected to an accelerated aging process, which included $468 \mathrm{~h}$ of thermal degradation in a convection oven at $50{ }^{\circ} \mathrm{C}$ and $40 \mathrm{~h}$ of photochemical degradation in an Atlas UVCON chamber, equipped with eight F40UVB lamps. A portion of these aged samples was washed at $85^{\circ} \mathrm{C}$ for $15 \mathrm{~min}$, in a solution of $\mathrm{NaOH}$ ( $1.0 \%$ by weight) and a surfactant (Triton $\mathrm{X}, 0.3 \%$ by weight), following the method proposed by Chariyachotilert et al. [24]. Finally, all samples were reprocessed by extrusion and compression in the above conditions.

According to the different recycling processes there were three different nanocomposites: PLAV-C30, which was not reprocessed, PLAR-C30, which was recycled without the washing step, and PLARW-C30 which was recycled with the washing step at $85{ }^{\circ} \mathrm{C}$. Before the characterization, all the samples were subjected to physical aging at room temperature for 3 weeks.

\section{Experimental Techniques}

Infrared spectra were recorded at a resolution of $4 \mathrm{~cm}^{-1}$, with a total of 16 scans, using a Nicolet iS10 spectrometer equipped with a diamond Attenuated Total Reflectance (ATR) accessory.

The diffraction patterns of the materials were obtained using a X'PERT-MPD diffractometer, equipped with a $\mathrm{CuK} \alpha$ generator $\left(\lambda_{1}=0.154056 \mathrm{~nm}\right.$ and $\left.\lambda_{2}=0.154439 \mathrm{~nm}\right)$ at $45 \mathrm{kV}$ and $40 \mathrm{~mA}$, in a $2 \theta$ range from 1.7 to $50^{\circ}$. The basal spacing of the clay was calculated using the mathematical expression of Bragg's law, given in Eq. (1):

$\lambda=2 d \times \sin \theta$

where $\lambda$ is the wavelength of the rays, $d$ is the spacing between the clay platelets and $\theta$ is the Bragg angle.

The intrinsic viscosity (IV) was measured in chloroform at $25 \pm 0.5{ }^{\circ} \mathrm{C}$ using an Ubbelohde viscosimeter. Intrinsic viscosity was obtained using the Kraemer equation [25], measuring four concentrations for each sample. The uncertainty was determined by linear regression with a $95 \%$ confidence level.

Differential scanning calorimetry (DSC) analysis was performed using a TA Instruments Q20 calorimeter on samples of 5-7 mg, in standard aluminum pans, at $5{ }^{\circ} \mathrm{C} /$ min under nitrogen atmosphere. The samples were first heated from 20 to $200{ }^{\circ} \mathrm{C}$, and then kept at $200{ }^{\circ} \mathrm{C}$ for 3 min to erase thermal history. After that, the samples were cooled to $0{ }^{\circ} \mathrm{C}$, and finally a second heating scan was performed until $200{ }^{\circ} \mathrm{C}$.

Thermogravimetric analysis was carried out using a TA Instruments TGA2050 thermobalance. Samples of 12-14 mg were heated at $10^{\circ} \mathrm{C} / \mathrm{min}$ from room temperature to $800{ }^{\circ} \mathrm{C}$ in dry nitrogen $\left(30 \mathrm{~cm}^{3} / \mathrm{min}\right)$.

The overall transmittance in the visible light region was measured according to the ISO 13468 standard, using a Shimadzu 2401 PC UV-Vis spectrophotometer equipped with a Shimadzu integrating sphere, using a scan speed of $200 \mathrm{~nm} /$ min.

The microhardness measurements were measured using a Type M Shimadzu microhardness tester equipped with a Vickers pyramidal indenter and applying a load of $25 \mathrm{~g}$ for $10 \mathrm{~s}$. Each measurement was repeated six times.

Water absorption was measured gravimetrically. Dried samples of $40 \times 25 \times 0.2 \mathrm{~mm}$ were dipped in flasks containing $100 \mathrm{ml}$ of $0.05 \mathrm{M}$ phosphate-buffered solution (PBS) at $\mathrm{pH} 7.4 \pm 0.2$. The flasks were located in an oven set at $37^{\circ} \mathrm{C}$. Specimens were removed at selected immersion periods, gently wiped and weighed, at room temperature, in a laboratory balance with a precision of $0.1 \mathrm{mg}$. The percentage of absorbed water at any time $t, M_{t}$, was determined by this expression:

$M_{t}(\%)=\frac{W_{t}-W_{0}}{W_{0}} \times 100$

where $W_{0}$ and $W_{t}$ denote, respectively, the weight of the specimens before and after the immersion in PBS. 
The permeability measurements were performed in a homemade permeation cell that has been described elsewhere [26]. The device consists of a permeation cell with two chambers, separated by a PLA membrane of known thickness. Nitrogen, oxygen and carbon dioxide permeability of the different samples has been determined at $30^{\circ} \mathrm{C}$ by means of diffusion experiments trough an initially purged membrane. After purging, a 0.2 MPa step variation of the pressure was imposed on the high-pressure side of the membrane, and the pressure on the low-pressure side was monitored. The permeability coefficient, $\mathrm{P}$, was estimated from the slope of pressure vs. time line after reaching steady state [27].

\section{Results and Discussion}

\section{Effects of Recycling Processes on the Structure}

The structure of PLA nanocomposites can be significantly altered by the degradation that takes place during the service life and the recycling process, which includes a melt reprocessing at high temperature and shear stress. On one hand, it is known that PLA and PLA-based materials are susceptible to thermal, photochemical and hydrolytic degradation processes that lead to a decrease of the average molecular mass, which can negatively affect the thermal, optical and mechanical properties of the material [28]. On the other hand, the dispersion of the nanoparticles in the polymer can be also altered during the recycling process due to the shear stress, resulting in a different morphology of the material, which also leads to changes in the properties.

The degree of dispersion was studied by means of XRD and TEM. Figure 1 shows the diffraction patterns of the PLA-C30 nanocomposite subjected to the different mechanical recycling processes, along with the pattern of pure $\mathrm{C} 30$. There were no differences in the region between 8 and $20^{\circ}$ (not shown), thus indicating that not important crystalline domains were formed in the polymer during the mechanical recycling of the nanocomposite. However, the changes observed at angles lower than $8^{\circ}$ reveal that the morphology of the composite was altered during the recycling processes.

Firstly, Fig. 1 shows the structure of the virgin nanocomposite. It can be seen that the characteristic (001) diffraction peak of the clay located at $2 \theta=4.8^{\circ}$, which corresponds to a basal spacing $\mathrm{d}_{001}=1.8 \mathrm{~nm}$, is located at lower angles in PLAV-C30 $\left(2 \theta=2.6^{\circ}\right)$, indicating the intercalation of the polymer chains within the galleries of the clay, and thus the formation of an intercalated structure. The nanocomposite also presents a broad second diffraction peak located at $2 \theta=5.4^{\circ}$, which may be related, at least partially, to the second-order reflection of the main diffraction. However, the formation of some clay aggregates with lower basal spacing

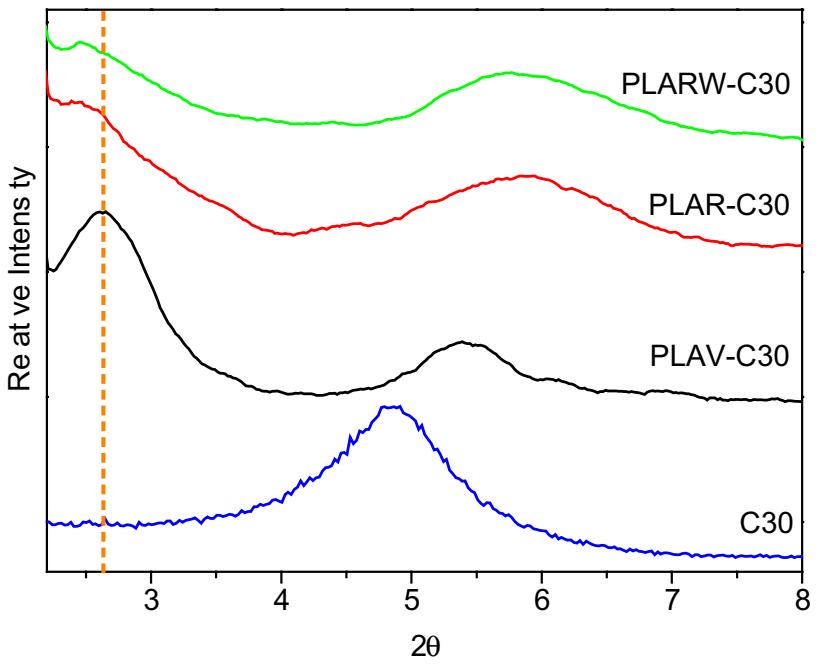

Fig. 1 XRD patterns of pure C30 and PLA-C30 nanocomposite subjected to different mechanical recycling processes ( $V$ virgin, $R$ recycled, $R W$ recycled with washing step)

during the melt processing could also contribute to this peak. A similar result was observed by Pluta et al. in PLA nanocomposites with $3 \mathrm{wt} \%$ of C30 [29].

Regarding the effect of the different mechanical recycling on the degree of dispersion in the nanocomposite, Fig. 1 shows that the (001) peak disappears almost completely in PLAR-C30 and PLARW-C30. The remaining weak (001) diffraction is displaced towards lower angles, which can be attributed to an improved dispersion of the clay, forming exfoliated structures in which the individual silicate layers are no longer close enough to interact with each other. These results are very important, since an improvement of the clay dispersion could lead to enhanced mechanical, optical and gas barrier properties. These results agree with those obtained by Scaffaro et al. in PLA nanocomposites with 5\% hydrotalcites, where an improvement of the clay dispersion was observed scanning electron microscopy (SEM) [23].

The effect of the recycling on the morphology can be also observed in the TEM micrographs corresponding to the virgin and recycled plastics (Fig. 2). It can be seen that, although some clay aggregates are still observed, the recycled material presents smaller particles than the virgin material, thus indicating and enhancement of the clay dispersion during the mechanical recycling, thus confirming the results observed in the XRD data.

The degradation of the polymer in the recycled materials was investigated by using intrinsic viscosity (IV) measurements, FTIR spectroscopy and differential scanning calorimetry.

The IV values are presented in Table 1. Firstly, it can be noticed that PLAR-C30, the nanocomposite recycled without the washing step, presents a reduction of around 

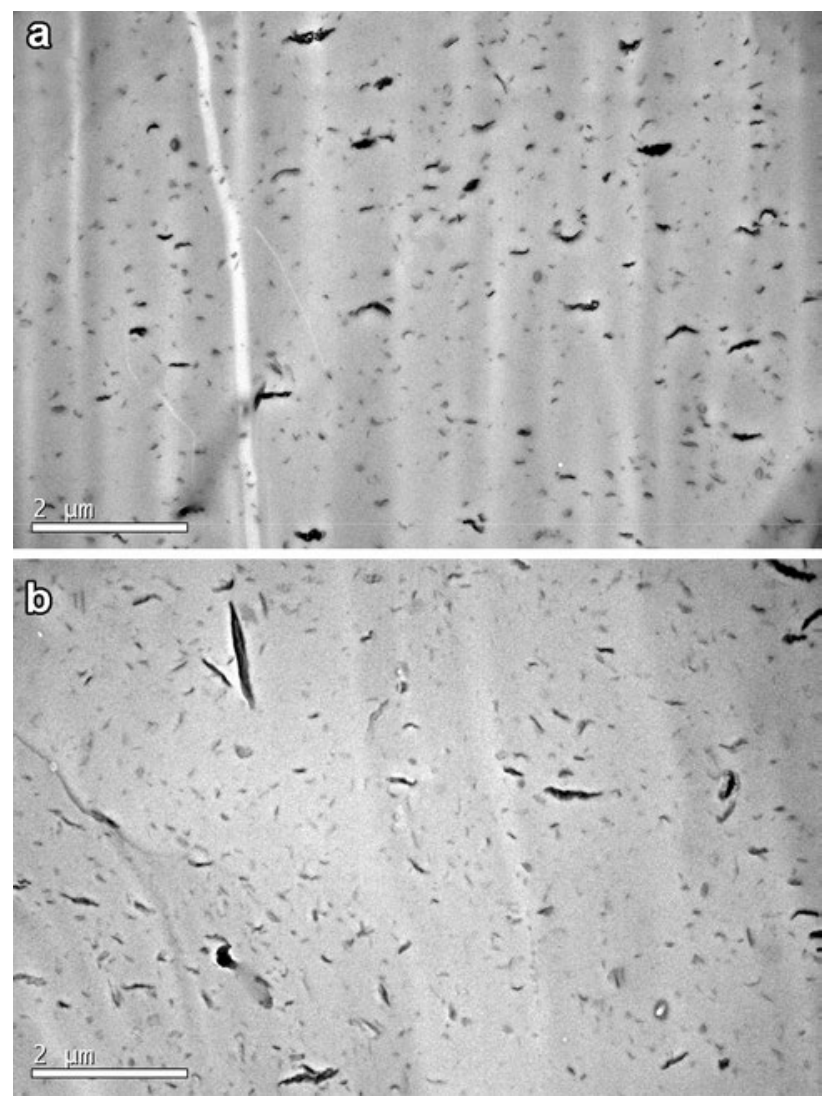

Fig. 2 TEM images of (a) PLAV-C30 and (b) PLAR-C30 ( $V$ virgin, $R$ recycled)

$7 \%$ on its intrinsic viscosity when compared with the virgin nanocomposite. This reduction in the intrinsic viscosity, and hence in the average molecular weight, is a consequence of chain scission processes in the polymer matrix due to the high temperatures and the shear stresses to which the polymer was subjected during the reprocessing, and could also be attributed to the degradation during the accelerated aging. Other authors have reported similar decreases in the molecular weight of unreinforced PLA samples subjected to a single reprocessing step $[17,18,24]$. This result is similar to that reported in neat PLA subjected to the same recycling process, in which a decrease of approximately $5 \%$ was obtained [1]. These results indicate that the clay does not have a very detrimental effect on the polymer degradation during the recycling of the nanocomposite. Moreover, the results reveal that the reduction of the intrinsic viscosity is small in this recycling process, so only limited effects on the mechanical and thermal properties of this recycled material must be expected.

Regarding the material subjected to the recycling process that included a washing step, PLARW-C30, Table 1 shows that the decrease of IV is more important in this case (near $20 \%$ when compared with the virgin nanocomposite). This result reveals that a demanding washing step such as the used in this work, with $\mathrm{NaOH}$ at high temperature, might play an important role on the degradation of PLA during its mechanical recycling, and thus could largely affect the properties of the recycled material. Again, unfilled PLA showed a very similar descent in the intrinsic viscosity, close to $20 \%$, when it was subjected to the recycling process including the washing step [1]. It was proposed that the washing in severe conditions weakened the structure of the polymer, generating acid groups that catalyzed the degradation during the following melt reprocessing.

The IV results indicate that the effects of the recycling strongly depend on the conditions selected for the recycling process. PLA-C30 nanocomposites can withstand a single recycling process without relevant decreases in the molecular weight of the polymer. However, the introduction of a demanding washing step in the recycling process promotes a further degradation of PLA during the reprocessing step, which might affect both the structure and final properties of the polymer.

After studying the effect of the different recycling processes on the molecular weight of PLA in the recycled nanocomposites with $\mathrm{C} 30$, the degradation suffered by the polymer was also studied by means of FTIR-ATR spectroscopy and DSC. The IR spectra (not shown) revealed that there are no major differences between the spectra of the studied materials. Although the IV values show the existence of chain scission during the recycling processes, which implies the formation of new carbonyl compounds, the IR spectra only show a minor difference in the carbonyl stretching band (centered at $1756 \mathrm{~cm}^{-1}$ ). This result is in good
Table 1 Properties of the virgin and recycled nanocomposites

\begin{tabular}{llllllll}
\hline Material & $\begin{array}{l}\text { Intrinsic } \\
\text { viscosity } \\
(\mathrm{mL} / \mathrm{g})\end{array}$ & $\Delta \mathrm{H}_{\mathrm{C}}(\mathrm{J} / \mathrm{g})$ & $\Delta \mathrm{H}_{\mathrm{M}}(\mathrm{J} / \mathrm{g})$ & $\mathrm{T}_{10}\left({ }^{\circ} \mathrm{C}\right)$ & $\mathrm{T}_{\max }\left({ }^{\circ} \mathrm{C}\right)$ & $\begin{array}{l}\text { Visible light } \\
\text { transmission } \\
(\%)\end{array}$ & $\begin{array}{l}\text { Vickers } \\
\text { hardness } \\
(\mathrm{MPa})\end{array}$ \\
\hline PLAV-C30 & $126 \pm 3$ & 27.0 & 29.2 & 340.0 & 367.3 & 83.4 & $189 \pm 5$ \\
PLAR-C30 & $117 \pm 4$ & 25.9 & 28.3 & 338.7 & 377.7 & 85.6 & $184 \pm 4$ \\
PLARW-C30 & $103 \pm 4$ & 27.7 & 28.2 & 339.4 & 374.1 & 85.9 & $180 \pm 4$ \\
\hline
\end{tabular}

The given uncertainties were determined with a $95 \%$ confidence level $V$ virgin, $R$ recycled, $R W$ recycled with washing step 
agreement with those reported by Badía et al. [18], which found that the generation of new carbonyl compounds during the reprocessing of PLA causes only a slight displacement of the carbonyl stretching band toward higher wavenumbers. Therefore, our results seem to indicate that, despite the degradation observed by means of intrinsic viscosity measurements, only small amounts of new carbonyl compounds are formed and the chemical structure of the polymer remains almost unchanged during the mechanical recycling.

DSC measurements can provide information about the structural changes of the polymer during the mechanical recycling of the nanocomposites. The first heating scans presented in Fig. 3 reveal that all the samples present a glass transition around $60{ }^{\circ} \mathrm{C}$, followed by an endothermic peak related to the densification of the amorphous regions of PLA, that is, the physical aging of the polymer [20]. The samples also show a cold crystallization peak $\left(\mathrm{T}_{\mathrm{cc}}\right)$ at approximately $110{ }^{\circ} \mathrm{C}$ and finally, a double peak melting endotherm $\left(\mathrm{T}_{\mathrm{m} 1}\right.$ and $\left.\mathrm{T}_{\mathrm{m} 2}\right)$ near $150^{\circ} \mathrm{C}$. The presence of this double melting peak has been explained as the result of a melt recrystallization process, which includes the melting of the less ordered crystals at a lower temperature $\left(\mathrm{T}_{\mathrm{m} 1}\right)$, their reorganization into more perfect structures and their subsequent melting at a higher temperature $\left(\mathrm{T}_{\mathrm{m} 2}\right)[30,31]$.

Concerning the recycling of the nanocomposite, it can be seen on Fig. 3 that PLAR-C30 shows a higher $\mathrm{T}_{\mathrm{cc}}$ than the virgin nanocomposite, while PLARW-C30 presents a lower value of $\mathrm{T}_{\mathrm{cc}}$. This behavior is different from that previously observed in the unreinforced polymer, where the mechanical recycling processes always led to lower $\mathrm{T}_{\mathrm{cc}}$ values in the recycled materials, which was explained as a consequence of the greater mobility of the shorter chains produced by the degradation during the recycling process $[1,21]$. The

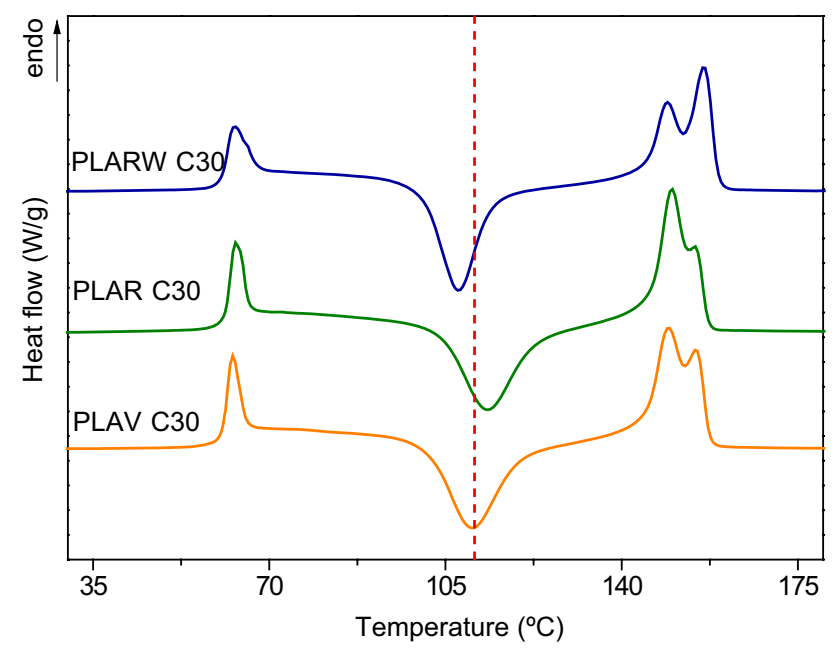

Fig. 3 DSC first heating scans of nanocomposites subjected to different mechanical recycling processes ( $V$ virgin, $R$ recycled, $R W$ recycled with washing step) different behavior observed in the recycled nanocomposites suggests that the mechanical recycling presents in this case two opposing effects on the crystallization behavior of the polymer. On the one hand, the generation of shorter polymer chains facilitates the crystallization of PLA, while, on the other hand, the improvement of the dispersion of the clay platelets (observed by means of XRD) hinders the packaging of the polymer chains, hence obstructing the formation of crystalline domains. In the recycling without the washing step (PLAR-C30), the degradation was small, as it was observed in the intrinsic viscosity measurements, thus prevailing the hindering effect of the better dispersion of the clay. However, the degradation observed when the demanding washing step was included (PLARW-C30) was significantly bigger, counteracting the effect of the better dispersion of the clay and thus decreasing the $\mathrm{T}_{\mathrm{cc}}$ of the material.

The presence of two opposite effects of the mechanical recycling in the nanocomposites is also noticeable in the shapes of the melting endotherms of the different materials. Figure 3 shows that PLAR-C30 presents a larger low temperature melting peak, which can be explained by the formation of less perfect crystalline structure and the hindering of the melt recrystallization mechanism, caused by the better dispersion of the clays in the polymeric matrix. On the other hand, PLARW-C30 shows a larger high temperature melting peak, due to the presence of shorter polymer chains, which can rearrange during the melting, forming more stable crystalline structures. Despite the changes observed in the $\mathrm{T}_{\mathrm{cc}}$ and melting endotherms, Fig. 3 shows that the crystallization and melting enthalpies are similar for each material (the values, measured as peak areas, are shown in Table 1), thus indicating that most of the crystals that melt during the heating are previously formed in the cold crystallization. This behavior suggests that all the materials are essentially amorphous, and that the different recycling processes do not cause significant changes on the crystallinity of PLA, thus supporting the results obtained from the XRD data. This conclusion is important, since the development of crystalline structures could affect the mechanical, optical and gas barrier properties of PLA. The fact that only marginal changes in crystallinity were observed during the different mechanical recycling processes might imply that various important properties of PLA remain unchanged despite the degradation of the polymer during the mechanical recycling.

\section{Properties of the Recycled Materials}

After studying the effects of the different recycling processes on the structure and morphology of the nanocomposite with C30, the effects of the structural changes on some of the properties of the recycled materials were analyzed.

The thermal stability was studied by means of TGA. Table 1 gives the values of. $\mathrm{T}_{10}$ and $\mathrm{T}_{\max }$, two characteristic 
temperatures commonly used as indicatives of the thermal stability of the materials, which are defined as the temperature at which $10 \%$ of the total mass is volatilized and the temperature of maximum rate of decomposition, respectively. Regarding the effect of the mechanical recycling on the thermal stability of the nanocomposite, it can be seen that both recycled materials present higher values of $\mathrm{T}_{\max }$, despite the degradation observed in the intrinsic viscosity measurements. This behavior can be related at least in part to the better dispersion of the clay platelets, observed in the XRD measurements, which effectively act as a barrier for the liberation of the decomposition products of PLA, thus increasing the characteristic temperatures of the recycled nanocomposites. Furthermore, the chain scission of PLA during the recycling produces a decrease of the average molecular weight of the polymer, which reduces the thermal stability, but also the generation of new carboxyl groups, which leads to stronger interactions between the polymer chains and, hence, increased thermal stability of the recycled materials.

The changes in the morphology reported in the previous section can result in changes in the optical properties of the materials, which play a key role in the field of food packaging. The light transmission of the different materials was studied by means of UV-Vis spectroscopy, according to the ISO 13468 standard. The results are shown in Fig. 4 and Table 1.

Figure 4 shows the UV-Vis spectra of neat PLA, and the virgin and recycled nanocomposites. When compared with neat polymer, nanocomposites present higher absorption in the UV region of the spectrum, which confirms that the incorporation of $\mathrm{C} 30$ provides some protection against the photochemical degradation caused by the UV radiation,

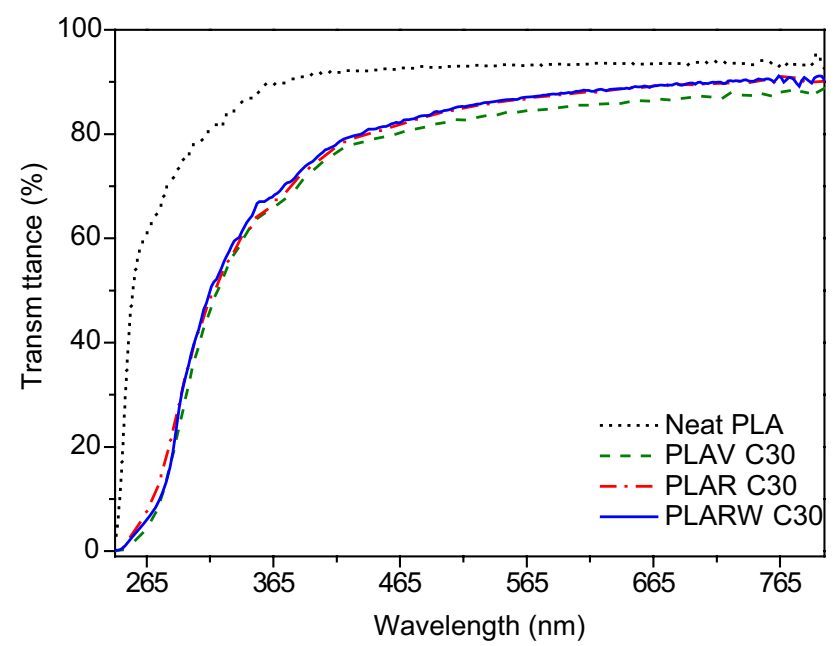

Fig. 4 UV-Vis spectra of the neat PLA and PLA-C30 nanocomposites subjected to different mechanical recycling processes ( $V$ virgin, $R$ recycled, $R W$ recycled with washing step) which may be interesting from the point of view of food packaging applications. Respecting the visible part of the spectrum, between 400 and $800 \mathrm{~nm}$, the addition of clays causes a decrease on the light transmission of the polymer, due to the presence of clay aggregates in the polymer matrix, which scatter and reflect the light, as it was pointed out by Cele et al. [32]. However, it is worth to note that both recycled nanocomposites present a higher visible light transmission than the virgin one. This result can be explained by the better dispersion of the clay nanoparticles in the recycled nanocomposites, observed by means of XRD and TEM, which reduces the amount of clay aggregates. Table 1 shows that the two recycled nanocomposites present good visible light transmission, higher than $85 \%$. In these materials, mechanical recycling not only does not worsen properties but even improves optical clarity.

The effect of the different recycling processes under consideration on the structure could also affect the mechanical properties and, hence, the potential use of recycled materials in various applications. In order to study the effects of the mechanical recycling, microhardness indentation tests were performed because it has been suggested that hardness measurement is more sensitive to molar mass changes than other mechanical characterization techniques [33]. The results that are shown on Table 1 point out that the different mechanical recycling processes causes a small decrease, lower than $10 \%$, in the Vickers hardness of the nanocomposite. The decrease of the hardness can be related to the lower molecular weight of PLA in the recycled materials [34]. However, the decrease is very small because the improved dispersion of the clay platelets in the recycled nanocomposites causes an increase on the hardness of the samples, thus partially counteracting the negative effect of the degradation of PLA during the different mechanical recycling processes. A similar behavior was observed by Scaffaro et al. in PLA/hydrotalcites nanocomposites, which showed an increase of the Young modulus even after three reprocessing steps [23]. The moderate impact of the recycling processes on the hardness seems to indicate that the mechanical properties of the recycled materials should not be a limiting factor when considering the use of recycled PLA based materials in packaging applications.

The permeability is one of the most important properties of the materials used in food packaging applications, because this property is one the main factors for determining the shelf life of the packed products. In this work, the effect of the aging and recycling processes on the permeability of the material to $\mathrm{O}_{2}, \mathrm{~N}_{2}$ and $\mathrm{CO}_{2}$ was evaluated. The results, shown in Fig. 5, indicate that the permeability of the nanocomposite clearly decreases as a result of the mechanical recycling. Again, this behavior must be explained by considering two opposing effects of the recycling processes. On one hand, the polymer degradation during the service life and the recycling process must favor the permeation, 


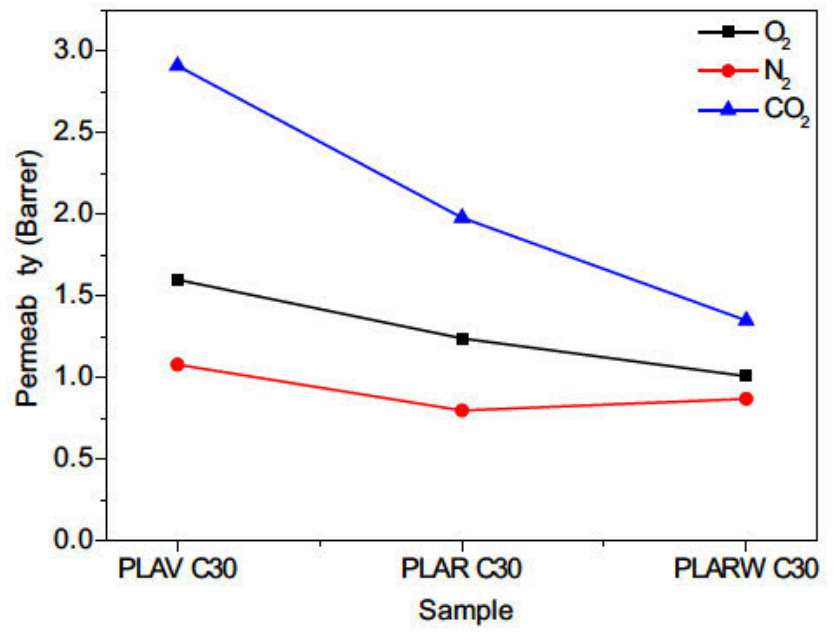

Fig. 5 Permeability of the virgin and recycled nanocomposites ( $V$ virgin, $R$ recycled, $R W$ recycled with washing step)

because fractional free volume, $v_{f}$, grows as a consequence of the molecular weight reduction and, according to Doolitle's equation, gas diffusion through a glassy polymer becomes easier if the $v_{f}$ increases [35]. On the other hand, XRD and TEM experiments indicate that the melt reprocessing improves the dispersion of the impermeable clay platelets into the polymer, thus increasing the tortuosity of the paths followed by the gas molecules when diffusing through the polymer, which leads to decreased permeability. In our case, the low permeability values corresponding to the recycled materials reveal that the improvement of the dispersion of the clay is the predominant effect of the recycling processes.

In summary, the results indicate that the mechanical recycling of PLA-C30 nanocomposites cause an improvement on the gas barrier properties as a consequence of the better dispersion of the clay due to the reprocessing.

Water absorption and hydrothermal degradation tests are widely used to measure the quality and degradability of materials [36]. In addition, these tests are especially important in materials such as those studied in this work, because it is likely for these materials to be in contact with liquids or wet products during their service life.

Figure 6 presents the water absorption curves of the virgin and recycled nanocomposites. It can be seen that for all materials, the amount of water absorbed does not reach equilibrium but grows continuously with the immersion time. This behavior was observed in a previous study with unfilled PLA [21], and was explained as the result of a two-stage sorption process. In the first stage, the fast water absorption is driven by the concentration gradient of the diffusant, while in the second stage the absorption is driven by the swelling and relaxation of the polymer [21]. Similar results have been reported by Davis et al. [21, 37]. Furthermore, the hydrolytic degradation of PLA after long immersion time

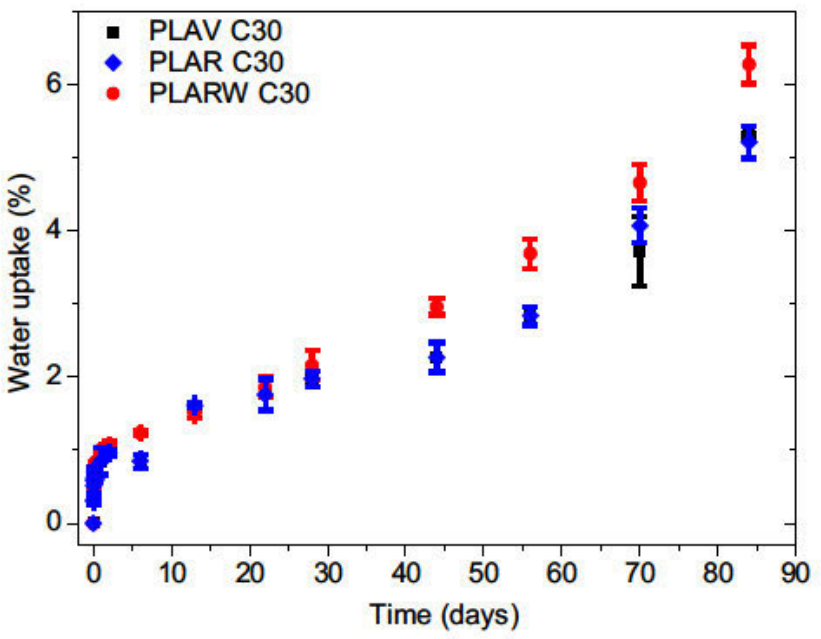

Fig. 6 Water absorption curves for PLAV-C30, PLAR-C30 and PLARW-C30 ( $V$ virgin, $R$ recycled without washing step, $R W$ recycled with washing step)

generates holes and hydrophilic products, which may facilitate the absorption of water [38]. Balart et al. also reported the appearance of cracks and holes in nanocomposites of PLA and hazelnut shell flour immersed in distilled water [36].

The complex nature of the sorption kinetics prevents the whole absorption process from being accurately described using a Fickian model, since this model assumes that the water transport is controlled by a concentration gradient and does not consider other phenomena such as the polymer degradation or the molecular relaxation. However, the first stage of the absorption process has great importance in practice for PLA-based materials, because an important fraction of these materials is used in the packaging of fresh food, so it would be interesting to elucidate if that first stage is controlled by a concentration gradient and can be accurately described using a Fickian model. An accurate model would allow the estimation of the water absorption parameters, and thus the study of the effect of the mechanical recycling on the kinetics of the first stage of the water absorption of the different samples.

For evaluating the accuracy of the Fickian model at short absorption times and calculating the parameters of the diffusion process, i.e., the diffusion coefficient and the moisture content at equilibrium, the experimental water absorption data for $120 \mathrm{~min}$ were fitted using a numerical solution of Fick's second law, given by Eq. (3) [39]:

$\frac{M_{t}}{M_{\infty}}=1-\frac{8}{\pi^{2}} \sum_{n=0}^{\infty} \frac{1}{(2 n+1)^{2}} \exp \left(\frac{-(2 n+1)^{2} \pi^{2} D t}{h^{2}}\right)$

where $M t$ is the moisture content at time $t, M_{\infty}$ is the moisture content at equilibrium or saturation mass, $D$ is the 
apparent diffusion coefficient and $h$ is the thickness of the sample. The serial expansion of Eq. (3) was truncated and only the first ten terms were considered. Finally, the values of $D$ were corrected by using Eq. (4), in order to consider the real dimensions of the samples used in the experiment, as indicated by Gupta and Pawar [40]:

$D c=D \times\left(1+\frac{h}{x}+\frac{h}{y}\right)$

where $D c$ is the corrected diffusion coefficient and $h, x$ and $y$ are the thickness, length and width of the samples, respectively.

The results obtained by fitting the experimental data using Eq. 3 are summarized in Fig. 7 and Table 2. It can be seen that, for short immersion times, the water absoprtion can be adequately fitted using Eq. 3, thus revealing that the diffusion of water is driven by the concentration gradient in the first stages of the absorption.

Table 2 and Fig. 7 reveal that virgin and recycled nanocomposites behave similarly during the first stages of the absorption process. Only the material obtained when the recycling process includes the washing step shows slightly higher values of the diffusion coefficient and the saturation water. In order to explain these results, the structural changes taking place during the recycling processes must be again considered. The degradation of the recycled materials, responsible for the decrease in the intrinsic viscosity that has been shown above, generates hydrophilic groups and an increase of free volume that favors the diffusion and absorption of water. However, the improved dispersion of clay nanoparticles slows the diffusion of water, so that recycling causes a negligible net effect on the absorption of water

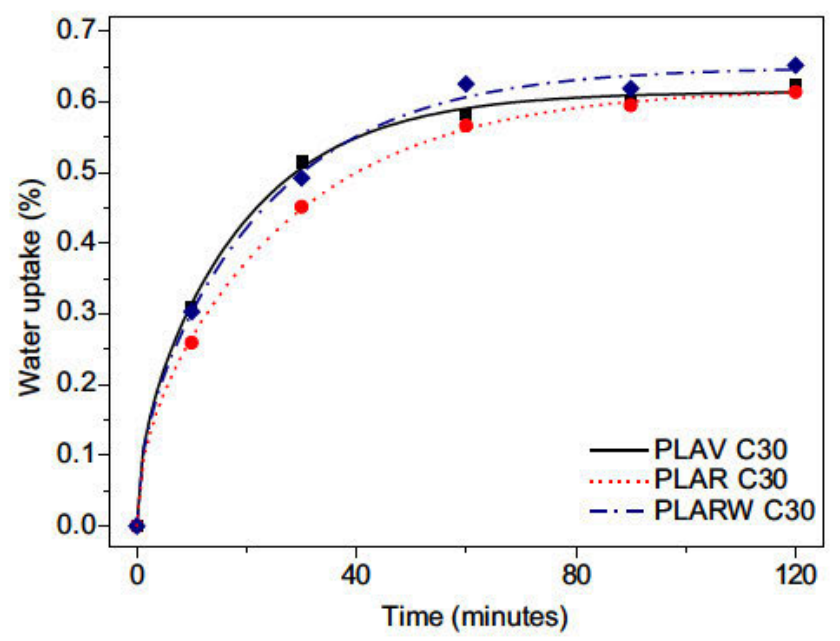

Fig. 7 Water absorption data, at short times, for the different samples. The lines correspond to the fitting of the data using Eq. 3 (with $\mathrm{n}=10)$ ( $V$ virgin, $R$ recycled, $R W$ recycled with washing step)
Table 2 Water absorption parameters calculated at short times for the different materials using Eq. 3 (with $\mathrm{n}=10$ )

\begin{tabular}{llll}
\hline Sample & $\mathrm{Dc} \times 10^{8}\left(\mathrm{~cm}^{2} / \mathrm{s}\right)$ & $\mathrm{M}_{\infty}(\%)$ & $\chi^{2}$ \\
\hline PLAV-C30 & $4.2 \pm 1.2$ & $0.61 \pm 0.01$ & 0.0001 \\
PLAR-C30 & $3.8 \pm 1.5$ & $0.62 \pm 0.01$ & 0.00003 \\
PLARW-C30 & $5.7 \pm 1.6$ & $0.65 \pm 0.01$ & 0.0002 \\
\hline
\end{tabular}

at short times. The slightly higher values of $D$ and $M_{\infty}$ in PLARW-C30 are explained as a consequence of the higher degradation observed in this material.

The difference in water absorption between the nanocomposites is higher in prolonged immersion times, as can be seen in Fig. 6. The increase in absorption at long immersion times in PLA-based materials has been related not only to the degradation of the polymer but also to a leaching process with the formation of pores and cracks [38]. Figure 6 shows that there is a different behavior between PLAR-C30 and PLARW-C30. The former shows a behavior very similar to that of the virgin nanocomposite, while the latter shows a slightly higher absorption. These results indicate that the polymer degradation during the accelerated aging, washing step and reprocessing favors the formation of pores and cracks at long immersion times. To determine if the initial degradation of the polymer also favors the decrease in the average molecular weight during the dipping, the intrinsic viscosity values were measured after different immersion times. The results shown in Fig. 8 indicate that the intrinsic viscosity decreases during immersion in water and that the decrease rate is similar in virgin and recycled nanocomposites.

The above results show that recycled nanocomposites have acceptable stability against hydrolytic degradation and

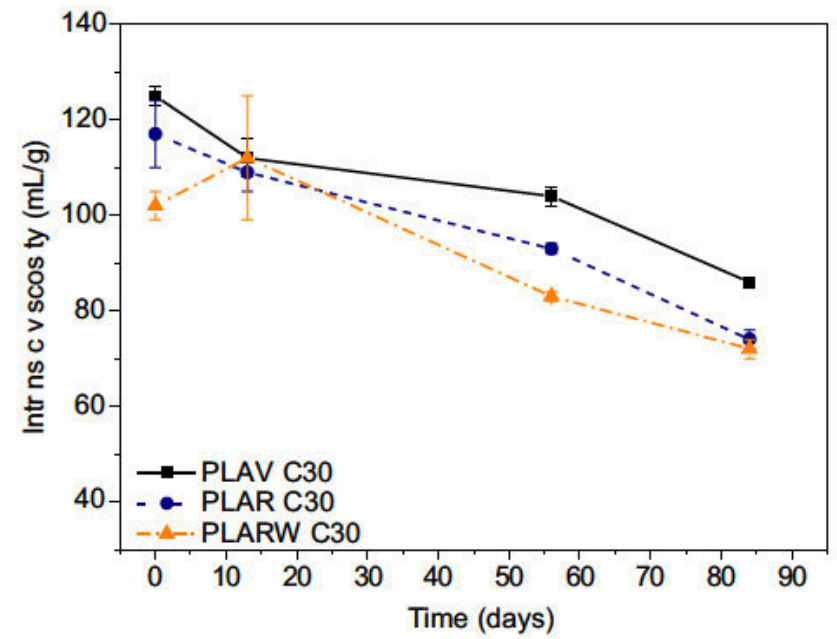

Fig. 8 Evolution of the intrinsic viscosity of the materials during the immersion ( $V$ virgin, $R$ recycled; $R W$ recycled with washing step) 
confirm that recycled nanocomposites have good properties, comparable to those of the virgin material, so they could be used in demanding applications.

\section{Conclusions}

The effects of accelerated aging and mechanical recycling processes on the structure and properties of PLA-clay nanocomposites have been studied. The results show two main differences between the structure of the recycled nanocomposites and that of the virgin. Firstly, mechanical recycling causes an improvement in the dispersion of the clay nanoparticles into the polymer, which has been observed by XRD and TEM. Secondly, intrinsic viscosity measurements reveal a decrease in the average molecular weight of the polymer in the recycled materials, especially when a demanding washing step is included during the recycling process. These two differences, which have also been observed in DSC curves, have opposite effects on the properties of recycled materials.

The virgin and recycled materials behave similarly in contact with water. The water absorption curves are complex, showing at least two distinct stages. In the first stage, at short immersion times, the absorption can be accurately described using a Fickian model. At long immersion times the absorption grows again due to the leaching and formation of voids and cracks in the polymer. The water absorption is slightly higher in the recycled material when the washing step is included in the recycling process, due to the higher molecular weight decrease in this case, which cannot be fully compensated by the better dispersion of the clay in the polymer. However, the decrease in intrinsic viscosity due to hydrolytic degradation is similar for virgin and recycled nanocomposites.

Despite the decrease of the molecular weight, the Vickers hardness remains almost unchanged and the thermal stability of the recycled nanocomposites is even slightly higher than that of the virgin, due to the better dispersion of the clay and the formation of carboxyl groups. The better dispersion of the clay also explains the higher optical clarity of the recycled nanocomposites, as well as the improved barrier properties for oxygen, nitrogen and carbon dioxide.

In general, the effect of accelerated aging and mechanical recycling processes on the properties of the nanocomposite is limited. The recycled nanocomposites have similar or even better properties in some cases than those of the virgin material, so the use of recycled nanocomposites in demanding applications, and even in the same applications as the virgin material, should not be ruled out.

Acknowledgements The authors would like to thank the Centro Nacional de Microscopía Electrónica and the CAI Difracción de Rayos X of the Universidad Complutense de Madrid (Spain), for the collaboration in the TEM and XRD measurements, respectively. The authors also would like to acknowledge the funding from MINECOSpain (project MAT2013-47972-C2-2-P), Universidad Politécnica de Madrid (project UPM RP 160543006) and Ecoembes (project DEHIPLA-R).

\section{References}

1. Beltrán FR, Lorenzo V, Acosta J, de la Orden MU, Martínez Urreaga $\mathbf{J}$ (in press) J Environ Manag

2. Badia JD, Santonja-Blasco L, Martínez-Felipe A, Ribes-Greus A (2012) Polym Degrad Stab 97:1881

3. Auras R, Lim L, Selke S. E. M., Tsuji H (2010) Poly(lactic acid): synthesis, structures, properties, processing, and applications. Wiley, New Jersey

4. Raquez J, Habibi Y, Murariu M, Dubois P (2013) Prog Polym Sci 38:1504

5. Rhim J, Park H, Ha C (2013) Prog Polym Sci 38:1629

6. Souza PMS, Morales AR, Marin-Morales M, Mei LHI (2013) J Polym Environ 21:738

7. Aeschelmann F, Carus M (2015) Ind Biotechnol 11:154

8. Reddy MM, Vivekanandhan S, Misra M, Bhatia SK, Mohanty AK (2013) Prog Polym Sci 38:1653

9. Leejarkpai T, Mungcharoen T, Suwanmanee U (2016) J Clean Prod 125:95

10. Mülhaupt R (2013) Macromol Chem Phys 214:159

11. Piemonte V (2011) J Polym Environ 19:988

12. Cosate de Andrade MF, Souza PMS, Cavalett O, Morales AR (2016) J Polym Environ 24:372

13. Rossi V, Cleeve-Edwards N, Lundquist L, Schenker U, Dubois C, Humbert S, Jolliet O (2015) J Clean Prod 86:132

14. Niaounakis M (2013) Biopolymers reuse, recycling, and disposal. William Andrew Publishing, Oxford

15. Badia JD, Ribes-Greus A (2016) Eur Polym J 84:22

16. Badia JD, Gil-Castell O, Ribes-Greus A (2017) Polym Degrad Stab 137:35

17. Scaffaro R, Morreale M, Mirabella F, La Mantia FP (2011) Macromol Mater Eng 296:141

18. Badia JD, Strömberg E, Karlsson S, Ribes-Greus A (2012) Polym Degrad Stab 97:670

19. Żenkiewicz M, Richert J, Rytlewski P, Moraczewski K, Stepczyńska M, Karasiewicz T (2009) Polym Test 28:412

20. Nascimento L, Gamez-Perez J, Santana OO, Velasco JI, Maspoch ML, Franco-Urquiza E (2010) J Polym Environ 18:654

21. Beltrán FR, Lorenzo V, de la Orden MU, Martínez-Urreaga J (2016) Polym Degrad Stab 133:339

22. Kozlowski MA, Macyszyn J (2013) In: Silvestre C, Cimmino S (eds) Recycling of nanocomposites in: polymer nanomaterials for food packaging. CRC Press, Florida, pp 313-336

23. Scaffaro R, Sutera F, Mistretta MC, Botta L, La Mantia FP (2017) Express Polym Lett 11:555

24. Chariyachotilert C, Joshi S, Selke SEM, Auras R (2012) J Plast Film Sheeting 28:314

25. Kraemer EO (1938) Ind Eng Chem 30:1200

26. Arranz-Andrés J, Lorenzo V, de la Orden MU, Pérez E, Cerrada ML (2011) J Membr Sci 377:141

27. Rutherford SW, Do DD (1997) Adsorption 3:283

28. Le Marec PE, Ferry L, Quantin J, Bénézet J, Bonfils F, Guilbert S, Bergeret A (2014) Polym Degrad Stab 110:353

29. Pluta M, Jeszka JK, Boiteux G (2007) Eur Polym J 43:2819

30. Di Lorenzo ML (2006) J Appl Polym Sci 100:3145

31. Yasuniwa M, Tsubakihara S, Sugimoto Y, Nakafuku C (2004) J Polym Sci B Polym Phys 42:25 
32. Cele HM, Ojijo V, Chen H, Kumar S, Land K, Joubert T, de Villiers MFR, Ray SS (2014) Polym Test 36:24

33. Pillin I, Montrelay N, Bourmaud A, Grohens Y (2008) Polym Degrad Stab 93:321

34. Lorenzo V, Pereña JM (1999) Curr Trends Polym Sci 4:65-76

35. Cohen MH, Turnbull D (1959) J Chem Phys 31:1164

36. Balart JF, Montanes N, Fombuena V, Boronat T, Sánchez-Nacher L (2017) J Polym Environ 1
37. Davis EM, Theryo G, Hillmyer MA, Cairncross RA, Elabd YA (2011) ACS Appl Mater Interfaces 3:3997

38. Deroiné M, Le Duigou A, Corre Y, Le Gac P, Davies P, César G, Bruzaud S (2014) Polym Degrad Stab 108:319

39. Crank J (1975) The mathematics of diffusion. Clarendon Press, Oxford

40. Gupta KM, Pawar SJ (2005) Mater Sci Eng 412:78 